\title{
Extraction of a quantitative reaction mechanism from linear sweep voltammograms obtained on a rotating disk electrode
}

\author{
E. Tourwé ${ }^{1}$, T. Breugelmans ${ }^{1,2}$, R. Pintelon ${ }^{3} \&$ A. Hubin ${ }^{1}$ \\ ${ }^{1}$ Vrije Universiteit Brussel, Department of Metallurgy, \\ Electrochemistry and Materials Science, Belgium \\ ${ }^{2}$ Hogeschool Antwerpen, Department of Industrial Sciences and \\ Technology - Chemistry, Belgium \\ ${ }^{3}$ Vrije Universiteit Brussel, Department of Fundamental Electricity and \\ Instrumentation, Belgium
}

\begin{abstract}
A new methodology to quantitatively determine the mass and charge transfer parameters of an electrochemical reaction is applied successfully in this work for the study of the reduction of ferricyanide to ferrocyanide at a platinum electrode, rotating at $1000 \mathrm{rpm}$. It is concluded that the reaction can be described by a simple electron transfer and the values of the charge transfer parameters are: $\alpha_{O x}=5.00 \mathrm{E}$ $01 \pm 8.75 \mathrm{E}-03, k_{o x}=1.39 \mathrm{E}-08 \pm 2.28 \mathrm{E}-09$ and $k_{r e d}=1.25 \mathrm{E}+00 \pm 2.01 \mathrm{E}-01$.
\end{abstract}

\section{Introduction}

The aim of a kinetic study of an electrochemical reaction with a relatively simple mechanism (only mass and charge transfer steps, no adsorption, chemical reactions, etc.) is the determination of its reaction mechanism and the quantification of its kinetic charge transfer parameters (rate constants and transfer coefficients) and mass transfer parameters (diffusion coefficients). Linear sweep voltammetry (LSV) in combination with a rotating disc electrode (RDE) is a powerful technique for providing information on the mechanism and kinetics of an electrochemical reaction. Previously we set-up a statistically founded method to model an electrochemical reaction and to determine its mass and charge transfer parameters quantitatively [1]. 
The method requires the proposition of an appropriate mechanism for the reaction under study and its mathematical translation into an expression that analytically describes the voltammogram. Powerful parameter estimation algorithms (maximum likelihood combined with Gauss-Newton and LevenbergMarquardt minimization methods) are used to adjust the values of these model parameters, in order to obtain a good agreement between experimental and modeled data. The values of the model parameters that give rise to the best match, characterize the system quantitatively. Moreover, the method provides error estimates of the obtained parameter values. It is however only after a statistical evaluation of the obtained results, that it is decided whether the model is able to describe the experiments or not.

The simplest electrochemical reactions, which can be found among the different kinds of electrode processes, are those where electrons are exchanged across the interface by flipping oxidation states of transition metal ions in the electrolyte adjacent to the electrode surface [2]. The electrode acts as the source or sink of electrons for the redox reaction and is supposed to be inert. The reduction of ferricyanide to ferrocyanide at a platinum electrode is described in literature [2-5] as an example of such a mechanism, i.e.

$$
F e(C N)_{6}^{3-}+e^{-} \leftrightarrow F e(C N)_{6}^{4-}
$$

Because no complex mechanism is expected to take place, this system is chosen here to evaluate the methodology that was set-up by our group previously [1].

Diverging values for the charge transfer parameters of this reaction are found in literature. The rate constants (defined w.r.t. the overpotential) range from $5 \times$ $10^{-4} \mathrm{~m} / \mathrm{s}[6,7]$, over $1 \times 10^{-3} \mathrm{~m} / \mathrm{s}$ [5], to $2.4 \times 10^{-3} \mathrm{~m} / \mathrm{s}$ [8]. Beriet and Pletcher [9] found values from $110^{-3}$ to $2.5 \times 10^{-3} \mathrm{~m} / \mathrm{s}$, using both steady state and rapid scan voltammetry. Literature values for $\alpha$ vary from 0.45 to $0.61[4,7]$. This work aims to determine the parameter values in a reliable, statistically founded manner and to provide error estimates on these values.

\section{Experimental}

\subsection{Composition of the electrolyte}

The following analytical reagents are used (all Merck p.a.): $\mathrm{K}_{4}\left[\mathrm{Fe}(\mathrm{CN})_{6}\right] .3 \mathrm{H}_{2} \mathrm{O}$, $\mathrm{K}_{3}\left[\mathrm{Fe}(\mathrm{CN})_{6}\right]$ and $\mathrm{KCl}$. Solutions are made with once-distilled and deionized water. A $1 \mathrm{M} \mathrm{KCl}$ solution is used as the supporting electrolyte and the concentrations of the electroactive components ferri/ferrocyanide are $0.005 \mathrm{M}$. In that way, a negligible migration flux, constant activity and diffusion coefficients of the electroactive species, a low electrolyte resistance and a uniform current distribution are aimed at. 


\subsection{Experimental set-up}

A typical three electrode set-up is used for the electrochemical experiments [2, 10]. The electrochemical cell contains a $\mathrm{Ag} / \mathrm{AgCl}$ reference electrode (SchottGeräte), a platinum rotating disk working electrode and a platinum grid as counter electrode. The RDE is made at our department by embedding a $4 \mathrm{~mm}$ diameter polycrystalline platinum rod in an insulating mantle of polyvinlidenefluoride. The electrode is rotated by an RDE control system of Autolab. The rotation speed is set to $1000 \mathrm{rpm}$. The voltammograms are measured using a high resolution galvanostat/potentiostat PGSTAT30 (Autolab Instruments) of Ecochemie, controlled by the GPES 4.8 software. The scan rate is taken constant at $1 \mathrm{mV} / \mathrm{s}$. The step potential is set to $0.00015 \mathrm{~V}$, and this way a maximum number of data points is measured.

All measurements are performed in a $200 \mathrm{ml}$ glass electrolytic cell, thermostatted at $25 \pm 0.5^{\circ} \mathrm{C}$ using a water jacket connected to a thermostat bath (Lauda RE304).

Prior to the measurements, the electrolyte is deoxygenated by bubbling with nitrogen gas (Air Liquide) for 10 minutes, while during the experiment a nitrogen blanket is maintained over the cell. This results in a substantial flattening of the reduction plateau of ferricyanide. Besides, the cell is always shielded from light in order to avoid the following photochemical decomposition of ferrocyanide [11]:

$$
\begin{aligned}
& \mathrm{Fe}(\mathrm{CN})_{6}^{4-} \stackrel{\text { light }}{\leftrightarrow} \mathrm{Fe}(\mathrm{CN})_{5}^{3-}+\mathrm{CN}^{-} \\
& \mathrm{CN}^{-}+\mathrm{H}_{2} \mathrm{O} \leftrightarrow \mathrm{HCN}+\mathrm{OH}^{-}
\end{aligned}
$$

\subsection{Electrode pretreatment}

The reproducibility of the measurements was strongly increased by means of applying the following standardized pretreatment of the electrode surface:

- mechanical polishing of the platinum electrode on a rotating disk (Struers DP10, on cloth), succesively using a diamond paste of $7 \mu \mathrm{m}$ and of $1 \mu \mathrm{m}$ (Struers);

- ultrasonic rinsing with deionized water followed by degreasing with chloroform, also in an ultrasonic bath (Elma model T470/H) for 2 minutes;

- before each experiment, the electrode potential was 4 times swept between +0.55 and $-0.45 \mathrm{~V}$ vs $\mathrm{Ag} / \mathrm{AgCl}$, at a scan velocity of $10 \mathrm{mV} / \mathrm{s}$ and a rotation speed of $1000 \mathrm{rpm}$.

It is reported in literature [12], that such a pretreatment removes oxide and trace contaminants from the Pt surface, while the $\mathrm{O}_{2}$ and $\mathrm{H}_{2}$ evolution reactions are avoided in this potential range.

Each experiment is performed on a freshly prepared electrode. 


\section{Results and discussion}

The method to determine the mechanism and the values of its characteristic parameters is founded on four building blocks [1].

The results of the experimental study These are the current/potential couples defining the polarization curve. The mean of 11 experiments that were performed under identical conditions is used for modeling.

The mathematical expression believed to explain the experimental results This expression is derived from the basic equations that describe what is happening during an electrochemical reaction. It is formulated based on a well-considered model for the studied reaction. It has the following form: current $=$ function (potential, experimental parameters, model parameters), where the experimental parameters describe the experimental conditions, like e.g. temperature, rotation speed of the RDE, concentration, ..., and the model parameters are the unknown parameters that need to be quantitatively determined, like e.g. rate constants, transfer coefficients, etc.

The fitting procedure In this block the differences between experimental and theoretical data are minimized. Therefore, a weighted least squares cost function $V_{W L S}$ is formulated:

$$
V_{W L S}=\frac{1}{2} \sum_{l=1}^{N_{d p}} \frac{\left(I_{m}(l)-I(l, \theta)\right)^{2}}{\sigma_{I}(l)^{2}}=\frac{1}{2} \epsilon^{T} \epsilon
$$

where:

- $N_{d p}$ : the number of data point in the experimental polarization curve

- $I_{m}(l)$ : the mean value of the measured current

- $\sigma_{I}$ : standard deviation on $I_{m}$, calculated from $M$ repeated measurements

- $\epsilon \in \mathbb{R}^{1 \times N_{d p}}$ : the error vector given by

$$
\epsilon=\left(\begin{array}{c}
\frac{I_{m}(1)-I(1, \theta)}{\sigma_{I}(1)} \\
\vdots \\
\frac{I_{m}(l)-I(l, \theta)}{\sigma_{I}(l)} \\
\vdots
\end{array}\right)
$$

- $I(l, \theta):$ the 'model' value of I

- $\theta$ : the model parameter vector

The Gauss-Newton and Levenberg-Marquardt method are implemented to minimize this cost function and eventually it provides the parameter values which best describe the data. Moreover, the standard deviation on these parameters is also calculated. 


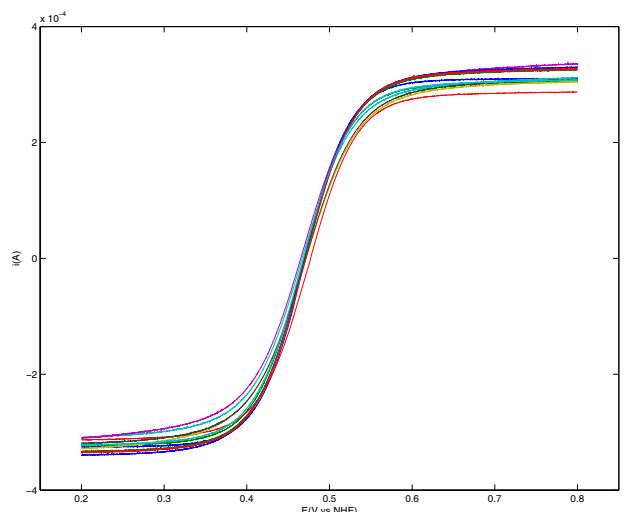

Figure 1: Voltammograms of the reduction/oxidation of $0.005 \mathrm{M}$ ferri/ferrocyanide in $1 \mathrm{M} \mathrm{KCl}$, at $1000 \mathrm{rpm}$.

A statistical evaluation If a statistical evaluation of the fitting results demonstrate a good description of the experiment by the model, a quantitative reaction mechanism is obtained. If, on the other hand, no good agreement between experiment and model is achieved, a new mechanism has to be proposed and the 2 previous steps should be repeated.

The results for each of these blocks are discussed in the following sections.

\subsection{Results of the experimental study}

The equilibrium potential of a $0.005 \mathrm{M}$ ferri/ferrocyanide solution in $1 \mathrm{M} \mathrm{KCL}$ equalled $0.470 \pm 0.001 \mathrm{~V} / \mathrm{NHE}$. As advised in [1], 11 polarization curves are measured under identical experimental conditions. The results are shown in figure 1.

From figure 1 it is seen that the current depends on the electrode potential for overvoltages of about $\pm 100 \mathrm{mV}$. At higher overvoltages, a limiting current is reached.

Figure 2 shows the mean experimental voltammogram and its $95 \%$ confidence interval $\pm 2 \sigma$, with $\sigma$ the standard deviation of the current, calculated from figure 1 .

\subsection{The mathematical expression believed to explain the experimental results}

For reaction (1) a mathematical expression that describes the polarization curves is derived, taking into account mass and charge transfer. The basic equations which are used, were described previously [1]. 


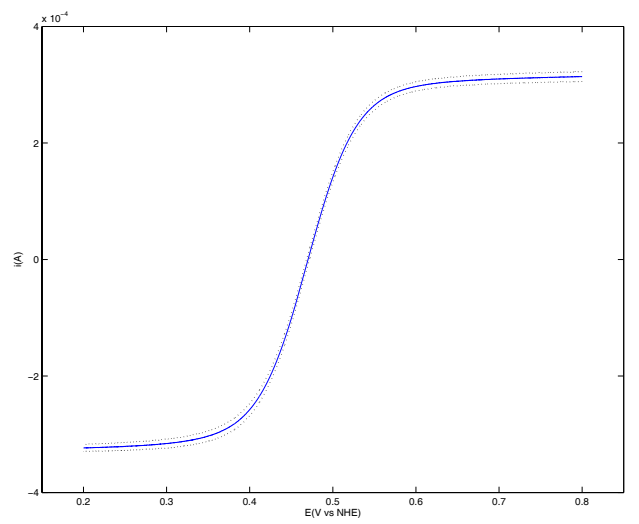

Figure 2: Mean voltammogram and its $95 \%$ confidence interval of the reduction/oxidation of $0.005 \mathrm{M}$ ferri/ferrocyanide in $1 \mathrm{M} \mathrm{KCl}$, at $2000 \mathrm{rpm}$.

The following expression for the current as a function of potential is obtained:

$$
i=\frac{n F S\left(K_{o x} c_{r e d}^{*}-K_{r e d} c_{o x}^{*}\right)}{1+n F S\left(\frac{K_{o x} c_{r e d}^{*}}{i_{l i m, o x}}-\frac{K_{r e d} c_{o x}^{*}}{i_{l i m, r e d}}\right)}
$$

with:

- $i$ : the current(in $A$ )

- $n$ : the number of electrons exchanged in the reaction

- $F$ : Faraday's constant $(96485 \mathrm{C} / \mathrm{mol})$

- $K_{o x}$ : the potential dependent rate constant for the oxidation half reaction, given by: $K_{o x}=k_{o x} \exp \frac{\alpha_{o x} n F E}{R T}$

- $K_{\text {red }}$ : the potential dependent rate constant for the reduction half reaction, given by: $K_{\text {red }}=k_{\text {red }} \exp \frac{-\alpha_{r e d} n F E}{R T}$

- $E$ : the potential (in $V$ vs NHE)

- $R$ : ideal gas constant $(8.32 \mathrm{~J} / \mathrm{molK})$

- $T$ : the absolute temperature (in $\mathrm{K}$ )

- $c_{\text {red }}^{*}$ : the bulk concentration of the reducing agent (ferrocyanide) (in $\mathrm{mol} / \mathrm{m}^{3}$ )

- $c_{o x}^{*}$ : the bulk concentration of the oxidizing agent (ferricyanide) (in $\mathrm{mol} / \mathrm{m}^{3}$ )

- $i_{\text {lim,ox }}$ : the oxidation limiting current (in A)

- $i_{\text {lim,red }}$ : the reduction limiting current (in A)

- $k_{o x}$ : the rate constant for the oxidation half reaction (in $\mathrm{m} / \mathrm{s}$ )

- $k_{r e d}$ : the rate constant for the reduction half reaction (in $\mathrm{m} / \mathrm{s}$ )

- $\alpha_{o x}$ : the transfer coefficient in the sense of the oxidation

- $\alpha_{\text {red }}$ : the transfer coefficient in the sense of the oxidation 


\subsection{The equations needed for the fitting procedure}

Assuming that the sum of $\alpha_{o x}$ and $\alpha_{r e d}$ equals one, a model for the current (equation 6) with 3 unknown parameters is obtained, viz. $\alpha_{o x}, k_{o x}$ en $k_{r e d}$.

Next to this expression for the current, the minimization algorithms require expressions for the derivatives of the current w.r.t. the unknown parameters in the Jacobian matrix. These are given by:

$$
\begin{aligned}
& \frac{\delta i}{\delta \alpha_{o x}}= \\
& \frac{n^{2} S E F^{2} i_{\text {lim }, o x}^{2} i_{\text {lim }, \text { red }}^{2} c_{r e d}^{*} \exp \frac{\alpha_{o x} E F n}{R T} k_{o x}}{R\left(c_{\text {red }}^{*} \exp \frac{\alpha_{o x} E F n}{R T} F i_{\text {lim,red }} k_{o x} n S+i_{\text {lim,ox }}\left(i_{\text {lim, red }}-c_{o x}^{*} \exp \frac{\left(-1+\alpha_{o x}\right) E F n}{R T} F k_{r e d} n S\right)\right)^{2} T} \\
& -\frac{n^{2} S E F^{2} i_{\text {lim,ox }}^{2} i_{\text {lim, red }}^{2} c_{o x}^{*} \exp \frac{\left(-1+\alpha_{o x}\right) E F n}{R T} k_{r e d}}{R\left(c_{\text {red }}^{*} \exp \frac{\alpha_{o x} E F n}{R T} F i_{\text {lim,red }} k_{o x} n S+i_{\text {lim,ox }}\left(i_{\text {lim,red }}-c_{o x}^{*} \exp \frac{\left(-1+\alpha_{o x}\right) E F n}{R T} F k_{r e d} n S\right)\right)^{2} T} \\
& \frac{\delta i}{\delta k_{o x}}= \\
& \frac{c_{r e d}^{*} \exp \frac{\alpha_{o x} E F n}{R T} F i_{l i m, o x} i_{l i m, r e d} n S\left(-i_{\text {lim }, o x} i_{l i m, r e d}\right)}{\left(c_{r e d}^{*} \exp \frac{\alpha_{o x} E F n}{R T} F i_{l i m, r e d} k_{o x} n S+i_{\text {lim,ox }}\left(i_{\text {lim,red }}-c_{o x}^{*} \exp \frac{\left(-1+\alpha_{o x}\right) E F n}{R T} F k_{r e d} n S\right)\right)^{2}} \\
& +\frac{c_{r e d}^{*} \exp \frac{\alpha_{o x} E F n}{R T} F i_{l i m, o x} i_{l i m, r e d} n S c_{o x}^{*} \exp \left(-1+\alpha_{o x}\right) E F n R T F\left(i_{\text {lim }, o x}-i_{\text {lim,red }}\right) k_{r e d} n S}{\left(c_{\text {red }}^{*} \exp \frac{\alpha_{o x} E F n}{R T} F i_{\text {lim,red }} k_{o x} n S+i_{\text {lim,ox }}\left(i_{\text {lim,red }}-c_{o x}^{*} \exp \frac{\left(-1+\alpha_{o x}\right) E F n}{R T} F k_{r e d} n S\right)\right)^{2}} \\
& \frac{\delta i}{\delta k_{r e d}}= \\
& \frac{c_{o x}^{*} \exp \frac{\left(-1+\alpha_{o x}\right) E F n}{R T} F i_{\text {lim }, o x} i_{\text {lim,red }} n S\left(-i_{\text {lim }, o x} i_{\text {lim }, \text { red }}\right)}{\left(c_{\text {red }}^{*} \exp \frac{\alpha_{o x} E F n}{R T} F i_{l i m, r e d} k_{o x} n S+i_{\text {lim,ox }}\left(i_{\text {lim,red }}-c_{o x}^{*} \exp \frac{\left(-1+\alpha_{o x}\right) E F n}{R T} F k_{r e d} n S\right)\right)^{2}} \\
& +\frac{c_{o x}^{*} \exp \frac{\left(-1+\alpha_{o x}\right) E F n}{R T} F i_{l i m, o x} i_{l i m, r e d} n S c_{r e d}^{*} \exp \frac{\alpha_{o x} E F n}{R T} F\left(i_{\text {lim,ox }}-i_{\text {lim,red }}\right) k_{o x} n S}{\left(c_{\text {red }}^{*} \exp \frac{\alpha_{o x} E F n}{R T} F i_{\text {lim,red }} k_{o x} n S+i_{\text {lim,ox }}\left(i_{\text {lim,red }}-c_{o x}^{*} \exp \frac{\left(-1+\alpha_{o x}\right) E F n}{R T} F k_{\text {red }} n S\right)\right)^{2}}
\end{aligned}
$$

\subsection{Fitting results and statistical evaluation}

To initiate the minimization procedure starting values are needed for the unknown model parameters and the values found in [13] are used for this purpose. The values for the other parameters (like temperature, bulk concentrations, etc.) can be found in the experimental section. Values for the limiting currents can be easily derived from figure 2 . The oxidation and reduction limiting currents are fixed to $3.1 \times 10^{-4} \mathrm{~A}$ and $-3.3 \times 10^{-4}$ A respectively.

At this point everything is ready to start the fitting procedure. The theoretical expression for the current (equation 6) will be fitted to the mean experimental polarization curve of figure 2, using the method described previously in [1].

The fitting results are illustrated in figure 3. Part (a) of this figure shows a comparison between the experimental polarization curve and a modeled curve, calculated with the best-fit-parameters. An exceptional agreement is obtained 

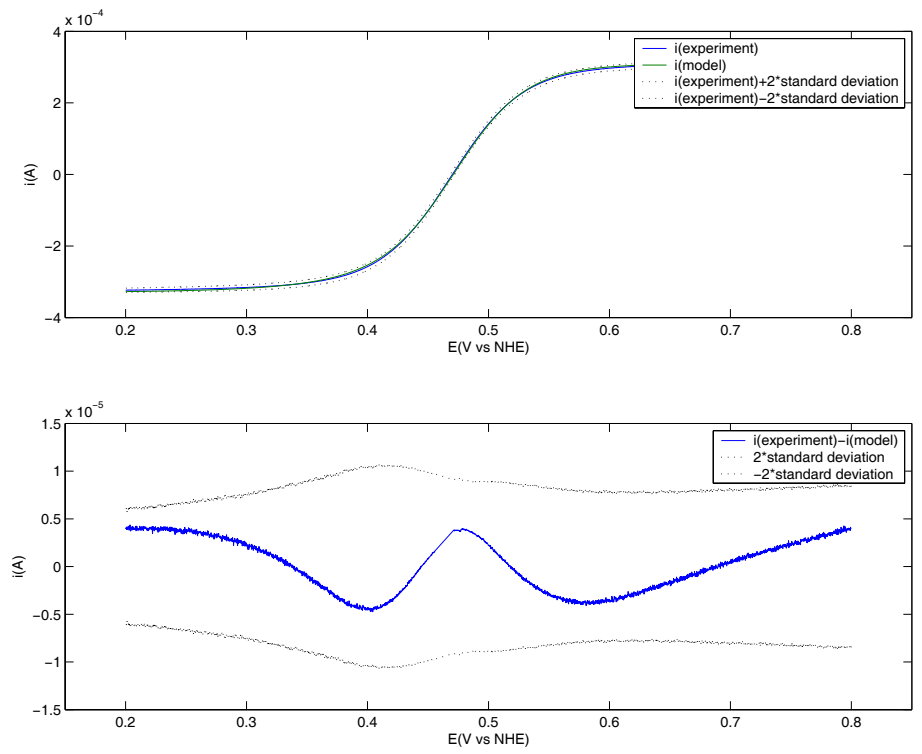

(a)

\begin{tabular}{|c|c|c|}
\hline parameter & estimated value & rel. std \\
\hline \hline$\alpha_{o x}$ & $6.16 \mathrm{E}-01$ & $3.87 \mathrm{E}-03$ \\
$k_{o x}$ & $1.52 \mathrm{E}-09$ & $4.05 \mathrm{E}-02$ \\
$k_{r e d}$ & $1.40 \mathrm{E}-01$ & $3.94 \mathrm{E}-02$ \\
\hline
\end{tabular}

(b)

\begin{tabular}{|c|c|}
\hline cost & condition number \\
\hline \hline $9.53 \mathrm{E}+02$ & $8.78 \mathrm{E}+01$ \\
\hline
\end{tabular}

(c)

Figure 3: Results of the fitting of equation 6 to the mean polarization curve at $1000 \mathrm{rpm}$ (3 parameter model): (a) comparison of the mean experiment with the model. (b) best-fit parameter vector and its relative standard deviation and (c) cost function and condition number.

and both curves are quasi indistinguishable. Also, the difference between the experimental and modeled data is plotted. It is observed that this difference lies in the confidence band, which is defined by \pm two times the standard deviation on the current, calculated from figure 1 . When performing multiple experiments, $95 \%$ of the experiments are expected to fall in this interval. It is therefore concluded that the model is able to describe the experiment appropriately. This is also evidenced 
Table 1: Best-fit parameter vector and its relative standard deviation, obtained when fitting the 5 parameter model to the mean polarization curve at $1000 \mathrm{rpm}$.

\begin{tabular}{|c|c|c|}
\hline parameter & estimated value & rel. std \\
\hline \hline$\alpha_{o x}$ & $5.00 \mathrm{E}-01$ & $8.76 \mathrm{E}-03$ \\
$k_{o x}$ & $1.39 \mathrm{E}-08$ & $8.19 \mathrm{E}-02$ \\
$k_{\text {red }}$ & $1.25 \mathrm{E}+00$ & $8.07 \mathrm{E}-02$ \\
$i_{\text {lim }, \text { ox }}$ & $3.11 \mathrm{E}-04$ & $5.79 \mathrm{E}-04$ \\
$i_{\text {lim,red }}$ & $-3.22 \mathrm{E}-04$ & $6.12 \mathrm{E}-04$ \\
\hline
\end{tabular}

by the low value of the cost function. The best-fit values for the the model parameters and their relative standard deviation are shown in part (b) of figure 3.

\subsection{A 5 parameter model}

The oxidation and reduction limiting currents are in fact also model parameter that need to be estimated. They replace the more obvious, intrinsic model parameters, viz. the diffusion coefficients of the oxidizing and reducing species. Previously the limiting currents were not considered as model parameters because good estimates are available from the experimental curves. However, it is preferred now to include them as model parameters because this way better estimates can be obtained. Consequently, a 5 parameter model is obtained and the expression for the Jacobian is adapted accordingly.

Again, an excellent match between modeled and experimental data is observed (figures not shown). The value of the cost function decreases from 9.53E+02 for the 3 parameter model to $1.42 \mathrm{E}+02$. This indicates an even better agreement between model and experiment. The values for the best-fit-parameters and their relative standard deviation are shown in table 1 . They differ slightly from those obtained for the 3 parameter model. As in the latter model the values for the limiting currents are not determined as accurately as in the 5 parameter model, the results of the 5 parameter model are considered as the best estimates.

It has to remarked that by performing these experiments at several rotation speeds of the RDE, the diffusion coefficients of the ferri- and ferrocyanide species can be calculated by the Koutecky-Levich method. As very accurate values for the limiting current are provided by this methodology, the diffusion coefficients will also be estimated accurately.

If the values for the rate constants are calculated w.r.t. the overpotential $\eta$ (e.g. $\left.K_{o x}=k_{o x}^{\prime} \exp \frac{\alpha_{o x} n F \eta}{R T}\right)$ instead of w.r.t. the potential one obtains the following values: $k_{o x}^{\prime}=1.9 \mathrm{E}-04$ and $k_{\text {red }}^{\prime}=1.9 \mathrm{E}-04$. These are of the same order of magnitude as the literature values [4-7,9]. 


\section{Conclusions}

A methodology to quantitatively determine the mass and charge transfer parameters of an electrochemical reaction that was proposed previously in [1] is validated and applied successfully in this work for the study of the redox couple ferri/ferrocyanide. It is concluded that the reaction mechanism is given by reaction (1) and the values of the charge transfer parameters are: $\alpha_{o x}=5.00 \mathrm{E}-01 \pm 8.75 \mathrm{E}-$ $03, k_{o x}=1.39 \mathrm{E}-08 \pm 2.28 \mathrm{E}-09$ and $k_{\text {red }}=1.25 \mathrm{E}+00 \pm 2.01 \mathrm{E}-01$.

\section{Acknowledgements}

Els Tourwé thanks the Flemish Institute for support of Scientific-Technological Research in Industry (I.W.T.).

\section{References}

[1] Tourwé, E., Pintelon, R. \& Hubin, A., Journal of Electroanalytical Chemistry, 594(1), pp. 50-58, 2006.

[2] Bamford, C. \& Compton, R., Electrode Kinetics: Principles and Methodology, volume 26 of Comprehensive Chemical Kinetics. Elsevier Science Publishers, 1986.

[3] Iwasita, T., Schmickelr, W., Hermann, J. \& Vogel, U. Journal of Electrochemical Society, 130, p. 2026, 1983.

[4] Angell, D. \& Dickinson, T. Journal of Electroanalytical Chemistry, 35, p. 55, 1972.

[5] Bruce, P., Lisowska-Oleksiak, A., Los, P. \& Vincent, C. Journal of Electroanalytical Chemistry, 367, p. 279, 1994.

[6] Jahn, D. \& Vielstich, W. Journal of Electrochemical Society, 109, p. 849, 1962.

[7] Tanaka, N. \& Tamamushi, R. Electrochimica Acta, 9, p. 963, 1964.

[8] Daum, P. \& Enke, C. Analytical Chemistry, 41, p. 653, 1969.

[9] Beriet, C. \& Pletcher, D. Journal of Electroanalytical Chemistry, 361, p. 93, 1993.

[10] Diard, J.P., Le Gorrec, B. \& Montella, C., Cinétique électrochimique. Hermann, 1996.

[11] Eisenberg, M., Tobias, C. \& Wilke, C. Journal of Electrochemical Society, 101, p. 306, 1954.

[12] Robertson, B., Tribollet, B. \& Deslouis, C. Journal of Electrochemical Society, 135, p. 2279, 1988.

[13] Vandeputte, S., Frequency response analysis of electrochemical systems at a rotating disk electrode under sinusoidal potentials or flow modulation. Ph.D. thesis, Vrije Universiteit Brussel, Brussel, Belgium, 1996. 